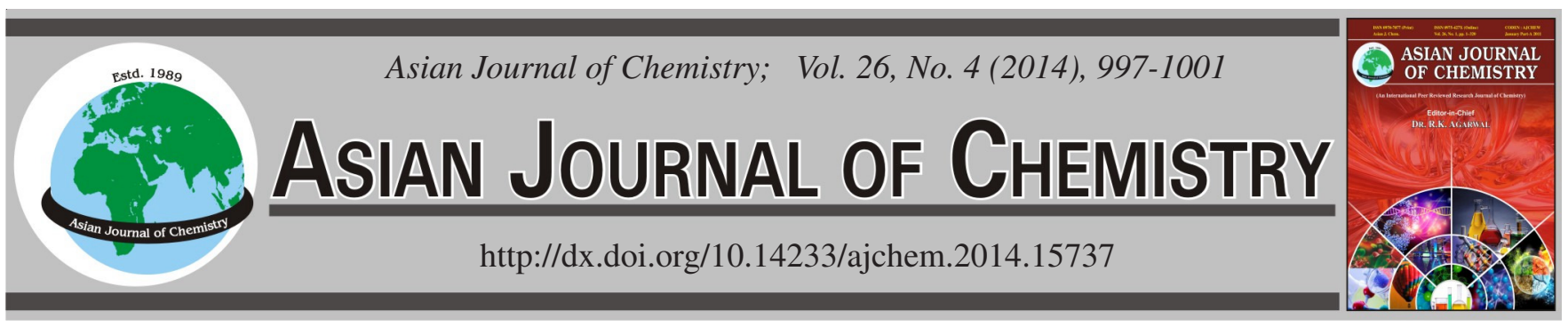

\title{
Determination of (-)-Borneol, Camphor and Isoborneol in Blumea balsamifera (L.) DC. Leaves by Simultaneous Ultrasonic and Microwave Assisted Extraction and Gas Chromatography
}

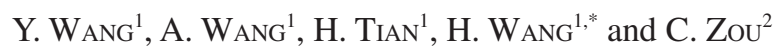

${ }^{1}$ School of Food Science and Technology, State Key Laboratory of Food Science and Technology, Jiangnan University, Wuxi 214122, P.R. China ${ }^{2}$ Gui Zhou Ai Yuan Eco-Pharmaceutical Development Co. Ltd., Luodian 550100, P.R. China

*Corresponding author: Fax: +86 510 85876799; Tel: +86 510 85917795; E-mail: xblack1983@eyou.com

\begin{abstract}
In present work, simultaneous ultrasonic and microwave assisted extraction followed by GC-FID was developed for quantitative analysis of the bioactive components of (-)-borneol, camphor and isoborneol in Blumea balsamifera leaves. After systematical investigation, the optimal experimental parameters microwave power $(100 \mathrm{~W})$ and extraction time $(30 \mathrm{~s})$ were investigated. The optimized method provided satisfactory linearity, precision, stability and recovery. The proposed method was applied for determination of three target compounds in leaves samples from 14 different times of harvest period. The variations of three target compounds were monitored and the result demonstrates (-)-borneol content of leaves was higher level from mid-October to next January. It has been shown that the proposed ultrasonic and microwave assisted extraction-GC-FID is a simple, rapid and reliable method for quantitative analysis of (-)-borneol, camphor and isoborneol in B. balsamifera leaves and a potential tool for quality assessment of B. balsamifera leaves.
\end{abstract}

Keywords: Blumea balsamifera (L.) DC., Leaves, Simultaneous ultrasonic and microwave assisted extraction, (-)-Borneol.

\section{INTRODUCTION}

Blumea balsamifera (L.) DC., locally called as "Ainaxiang" in Luodian China, is a traditional Miao nationality medicinal plant and belongs to the Family Asteraceae ${ }^{1}$. B. balsamifera has been reported to possess to promote digestion, reduce phlegm, antispasmodic and sudorific properties ${ }^{2,3}$. Chinese Pharmacopoeia defines that $B$. balsamifera leaves are the only plant source to extract natural (-)-borneol (Chinese name: Aipian $)^{4}$ and natural (-)-borneol is widely used in cardiovascular Chinese patent medicines ${ }^{5-9}$. In the production of "Aipian", the high quality raw leaves were selected to ensure high productivity. According to experience of herbalist, B. balsamifera leaves are harvested in autumn and winter, but the accurate and detailed (-)-borneol contents of leaves in growth period are not measured ${ }^{10}$. Therefore, the variation of (-)-borneol content must be monitored in harvest period. Meanwhile, "Aipian" contains not only (-)-borneol but also two impurities (isoborneol and camphor), so that their contents also need to be monitored. To the best of our knowledge, no report has yet appeared on the relevant investigation. To the above objective, we must rapidly and accurately determine (-)-borneol, isoborneol and camphor contents of leaves. (-)-Borneol, isoborneol and camphor are volatile compounds, the determination need to be performed by $\mathrm{GC}^{11}$ and there are a lot of alternative sample pretreatment methods. Previously, Zhang et $a l .{ }^{12}$ and Deng et al. ${ }^{13-15}$ have been used some pretreatment techniques (SD, MAE, UAE, MAE-HS-SPME and PHWEHS-SPME) for determining borneol and camphor content of Flos Chrysanthemi indici, Chrysanthemum indici and Amomum villosum lour.

Recently, simultaneous ultrasonic and microwave assisted extraction (UMAE) technique was developed and it is a complementary technique combining ultrasonic assisted extraction (UAE) and microwave assisted extraction (MAE) ${ }^{16}$. Ultrasonic energy allows greater penetration of solvent into the herbal tissue, increases the contact surface area and generates cavitation effect, thus improving the extraction efficiency ${ }^{17}$. Microwave can achieve instantaneous heating, thus reduce extraction time and accelerate the extraction ${ }^{18}$. Therefore, UMAE enables a significant reduction in the extraction time and the consumption of organic solvent ${ }^{19}$.

In accordance with the target of quality monitor and mastering variation of composition, a rapid, easy operation and reliable pretreatment technique is needed. The analysis speed is very important to quality monitor and production feedback. In the work, simultaneous ultrasonic and microwave assisted extraction (UMAE) is developed for quantitative analysis of (-)-borneol, isoborneol and camphor in Blumea balsamifera leaves. The experimental parameters were studied 
and the method precision, stability, recovery and linearity were investigated. The method was tested by the application to the determination of (-)-borneol, isoborneol and camphor in $B$. balsamifera leaves samples from 14 different times in harvest period.

\section{EXPERIMENTAL}

B. balsamifera leaves were collected at fourteen different times from 15th September 2011 to 30th March 2012 (interval of half a month, consecutively numbered from 1 to 14) from plants growing in wild surroundings in Luodian County (Southwest China), localised at $800 \mathrm{~m}$ altitude, $25^{\circ} 04^{\prime} \mathrm{N}$ and $106^{\circ} 28^{\prime}$ E. The plant was identified by Prof. Y.N. He (Institute of Biotechnology, Guizhou Academy of Agricultural Sciences, China) and a voucher specimen (CGA-Dafengai-Guizhou2009-11) was deposited in the Institute of Biotechnology, Guizhou Academy of Agricultural Sciences. After being collected, leaves were air-dried in the room temperature and then processed for powder with particle size of 80 mesh.

Standard (-)-borneol (97.0 \%) was purchased from SigmaAldrich (Shanghai, China), naphthalene (99.5\%) and analytical grade ethyl acetate and diethyl ether anhydrous were purchased from Sinopharm Chemical Reagent Co. Ltd. (Shanghai, China). Stock solutions $(10.0 \mathrm{mg} / \mathrm{mL})$ of the standard were prepared by dissolving (-)-borneol into ethyl acetate. Naphthalene was used as the internal standard (IS) in the study, for it was not contained in leaves. The internal standard solution $(10 \mathrm{mg} / \mathrm{mL})$ was made in ethyl acetate.

Optimization of simultaneous ultrasonic and microwave assisted extraction: Microwave power and extraction time might mainly affect the extraction efficiency. The effectiveness of microwave powers $(100-700 \mathrm{~W})$ and extraction times (30-360 s) were investigated. A simultaneous ultrasonic and microwave extraction apparatus model CW-2000 (Shanghai Xintuo Analytical Instruments Co. Ltd., China) was used in UMAE. $1 \mathrm{~g}$ sample and $10 \mathrm{~mL}$ ethyl acetate were put into a $100 \mathrm{~mL}$ vessel. Extraction process was performed in the apparatus chamber with an invariable ultrasonic power of $50 \mathrm{~W}$ and the vessel was then cooled to room temperature. The extraction solution was filtered and the filtrate was collected into $25 \mathrm{~mL}$ volumetric flask for GC-FID analysis.

GC-FID: GC analysis was performed using a gas chromatograph (Shimadzu-2010 Plus, Tokyo, Japan) with AOC20i autosamplers and a flame ionization detection system (FID). Data were collected by the GC solution software (Shimadzu). The gas chromatograph was equipped with a CP-WAX capillary column $(30 \mathrm{~m} \times 0.32 \mathrm{~mm} \times 0.22 \mu \mathrm{m})$. The injection port was held at $250{ }^{\circ} \mathrm{C}$ and used in split mode with a split ratio of 1:5. The detector temperature was $250^{\circ} \mathrm{C}$. Pure nitrogen (99.999\%) was used as the carrier gas at constant flow rate of $3 \mathrm{~mL} / \mathrm{min} ; \mathrm{H}_{2}$ : $47.0 \mathrm{~mL} / \mathrm{min}$; air: $400 \mathrm{~mL} / \mathrm{min}$. The oven temperature was initiated at $80^{\circ} \mathrm{C}$ (held for $5 \mathrm{~min}$ ), then raised at the rate of $10^{\circ} \mathrm{C} / \mathrm{min}$ to $220^{\circ} \mathrm{C}$, held for $5 \mathrm{~min}$ at this temperature.

Method validations: Linearity and repeatability of GC chromatogram system were verified. To obtain the linearity, replicate analyses of calibration solutions (from 0.1 to 2000 $\mu \mathrm{g} / \mathrm{mL}$ ) spiked with $1000 \mu \mathrm{g} / \mathrm{mL}$ naphthalene (IS) were directly analyzed with the GC-FID system. The repeatability was presented as the RSDs of retention times (RTs) $(n=6)$ of three target compounds. The method precision, stability, recovery, limit of detection (LOD) and limit of quantitation (LOQ) of UMAE were validated. No. $1 \mathrm{~B}$. balsamifera leaves sample was used as tested sample and it was harvested at 15 th September 2011. UMAE method precision was studied by eight replicate analyses of three target compounds in tested sample. The stability was studied by determining three target compounds of the tested sample every $4 \mathrm{~h}$ from 0 to $48 \mathrm{~h}$. The relative standard deviation (RSD \%) was calculated on the basis of the peak areas. The recovery was investigated by adding different volume gradient $(50,100$ and $150 \%$ of objective compound contents) of standard stock solution $(10 \mathrm{mg} / \mathrm{mL})$ and $1 \mathrm{~mL}$ of internal standard solution $(10 \mathrm{mg} / \mathrm{mL})$ to the extraction solutions $(10 \mathrm{~mL})$ of sample $(1 \mathrm{~g})$ containing known amounts of (-)-borneol $(5.5 \mathrm{~g} / \mathrm{kg})$, isoborneol $(0.016 \mathrm{~g} / \mathrm{kg})$ and camphor $(0.12 \mathrm{~g} / \mathrm{kg})$. LOD and LOQ were defined and determined as the minimum detectable amounts of three target compounds with a signal-to-noise ratio $(\mathrm{S} / \mathrm{N})$ of $3: 1$ and 10:1, respectively. Triplicate measurements were performed by UMAE methods.

Conventional reference extraction method: Hydrodistillation-solvent extraction (HDSE) was a conventional reference extraction method. Twenty grams of B. balsamifera leaves were carefully weighed and put into a $2 \mathrm{~L}$ distillation flask, $1 \mathrm{~L}$ distilled water was added and distilled (which was optimized as the best material/water ratio). $300 \mathrm{~mL}$ aqueous solution was collected at the end of hydrodistillation (the optimal condition according to our preliminary experiments). The solution was extracted with $300 \mathrm{~mL}$ diethyl ether anhydrous and then the diethyl ether anhydrous was concentrated to obtain the crude extract in a rotary evaporator under reduced pressure. The crude extract was dissolved in ethyl acetate for GC-FID analysis. All samples were prepared and analyzed in triplicate.

Separation, purification and identification of (-)-borneol: (-)-Borneol was separated through sublimation and purified by recrystallization. The crude extract (HDSE) was heated under $110^{\circ} \mathrm{C}$ and sublimated to collect impure (-)-borneol. The impure (-)-borneol were dissolved in petroleum ether $\left(60-90{ }^{\circ} \mathrm{C}\right)$ to obtain high-purity (-)-borneol by recrystallization. The optical activities of purified (-)-borneol and standard (-)-borneol were identified by NMR (Bruker AVANCE III 400 MHz Digital NMR Spectrometer, Bruker, Swiss) and WZZ-2A automatic polarimeter (INESA, China). The experiment was repeated six times and the average was calculated.

\section{RESULTS AND DISCUSSION}

Identification of (-)-borneol from leaves: CP-WAX capillary column of GC was not suitable for separating optical isomers, so that the separated (-)-borneol from B. balsamifera leaves needed to be identified by NMR and optical activity. The NMR spectral data are listed as follows: ${ }^{1} \mathrm{HNMR}\left(\mathrm{CDCl}_{3}\right.$,

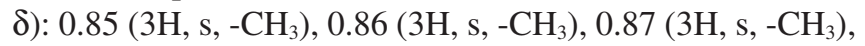
0.95 (1H, dd, H-6b), 1.25 (2H, m, H-5b, H-6a), 1.62 (1H, t, -OH), $1.70(2 \mathrm{H}, \mathrm{m}, \mathrm{H}-3 \mathrm{~b}, \mathrm{H}-5 \mathrm{a}), 1.90$ (1H, m, H-3a), 2.28 (1H, m, $\mathrm{H}-4), 4.04$ (1H, m, H-2). ${ }^{13} \mathrm{CNMR}\left(\mathrm{CDCl}_{3}, \delta\right): 13.4$ (C-10), 18.9 (C-8), 20.5 (C-9), 26.3 (C-6), 28.5 (C-5), 39.2 (C-3), 45.2 (C-4), 48.3 (C-7), 49.9 (C-1), 77.7 (C-2). The optical activities of separated (-)-borneol and standard (-)-borneol were determined by polarimeter and their specific rotations 
were $-35.0^{\circ}$ and $-35.1^{\circ}$ (ethanol, $20^{\circ} \mathrm{C}$ ), which showed that the separated (-)-borneol was levoisomer. The NMR spectral data and specific rotation verified that the separated (-)-borneol was actually (-)-borneol.

Optimization of simultaneous ultrasonic and microwave assisted extraction: The effects of the microwave power and extraction time were investigated in the study. $1 \mathrm{~g}$ No $1 B$. balsamifera leaves were treated through the UMAE method described above. The extraction efficiency of (-)-borneol, camphor and isoborneol in the leaves was investigated and the analytical results were compared to obtain the optimum extraction conditions, which is obtained by GC-FID measurement under different conditions.

Selection of microwave power: The experiments were carried out by extracting (-)-borneol, camphor and isoborneol from $B$. balsamifera leaves with four microwave powers (100 $\mathrm{W}, 200 \mathrm{~W}, 400 \mathrm{~W}$ and $700 \mathrm{~W}$ ) under the conditions of the 1:10 ratio of sample/solvent ratio $(\mathrm{g}: \mathrm{mL})$, the ultrasonic power of $50 \mathrm{~W}$ and 1 min extraction. The results are shown in Fig. 1a and we can find that the microwave power of $100 \mathrm{~W}$ has the best extraction efficiency for (-)-borneol, camphor and isoborneol. Therefore, $100 \mathrm{~W}$ was chosen as the best microwave power in the following extraction experiments.

Selection of extraction time: The influence of extraction time is shown in Fig. 1b on the extraction efficiency of (-)- borneol, camphor and isoborneol from B. balsamifera leaves. It can be seen that the best extraction efficiency was achieved at $30 \mathrm{~s}$. Considering the above results, the optimum extraction conditions were microwave power of $100 \mathrm{~W}$ and an extraction time of $30 \mathrm{~s}$.

Validation of method: Several levels of standard solutions (from 0.1 to $2000 \mu \mathrm{g} / \mathrm{mL}$ ) spiked with $1000 \mu \mathrm{g} / \mathrm{mL}$ naphthalene (IS) were directly analyzed with the GC-FID system. The linear range was $0.6-1875 \mu \mathrm{g} / \mathrm{mL}$ for (-)-borneol, isoborneol and camphor. The calibration curve for quantifying (-)-borneol was: $\mathrm{Y}=0.863 \mathrm{X}-0.0094$, with a correlation coefficient of 0.9992 (Y: Peak area ratio of (-)-borneol to IS, X: concentration ratio of (-)-borneol to IS), for isoborneol was: $\mathrm{Y}=0.9004 \mathrm{X}-$ 0.0096 , with a correlation coefficient of 0.9992 (Y: Peak area ratio of isoborneol to IS, X: concentration ratio of isoborneol to IS) and for camphor was: $\mathrm{Y}=0.8428 \mathrm{X}-0.0093$, with a correlation coefficient of 0.9991 (Y: Peak area ratio of camphor to IS, X: concentration ratio of camphor to IS). The repeatability of the chromatographic system was expressed by RSDs of three target compounds retention times and the RSDs ranged from 0.1 to $0.5 \%$. The results of UMAE method validation were shown in Table-1. The precision and stability of UMAE method were measured by RSD. The recoveries for (-)-borneol, camphor and isoborneol were 94, 89 and $87 \%$, respectively. LODs (from 0.16 to $0.21 \mu \mathrm{g} / \mathrm{mL}$ ) and LOQs (from 0.54 to
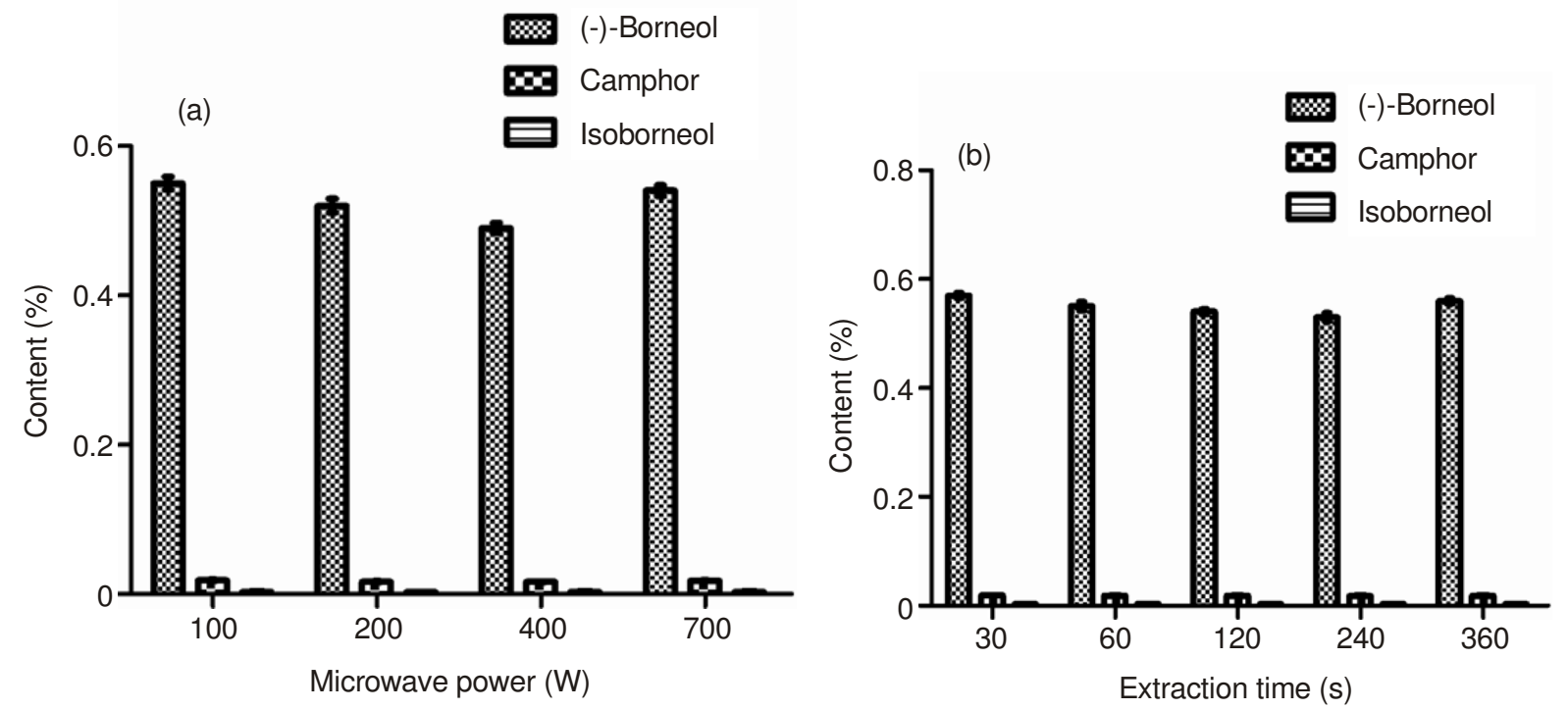

Fig. 1. Effect of microwave power (a) and extraction time (b) on the UMAE extraction efficiencies of (-)-borneol, camphor and isoborneol in Blumea balsamifera leaves

TABLE-1

PRECISION, STABILITY, RECOVERY AND DETECTION LIMIT

\begin{tabular}{|c|c|c|c|c|c|c|}
\hline \multirow{2}{*}{ Methods } & \multicolumn{2}{|c|}{ Recovery } & \multirow{2}{*}{ Precision RSD (\%) } & \multirow{2}{*}{ Stability RSD (\%) } & \multirow{2}{*}{$\mathrm{LOD}(\mu \mathrm{g} / \mathrm{mL})$} & \multirow{2}{*}{$\mathrm{LOQ}(\mu \mathrm{g} / \mathrm{mL})$} \\
\hline & Value $(\%)$ & RSD (\%) & & & & \\
\hline \multicolumn{7}{|c|}{ (-)-Borneol } \\
\hline UMAE & 94 & 4.1 & 4.1 & 3.1 & 0.16 & 0.54 \\
\hline HDSE & 98 & 3.6 & 3.3 & 4.5 & 0.16 & 0.54 \\
\hline \multicolumn{7}{|c|}{ Camphor } \\
\hline UMAE & 89 & 4.3 & 3.2 & 3.8 & 0.21 & 0.7 \\
\hline HDSE & 105 & 14.2 & 2.9 & 3.6 & 0.21 & 0.7 \\
\hline \multicolumn{7}{|c|}{ Isoborneol } \\
\hline UMAE & 87 & 5.3 & 2.9 & 3.4 & 0.16 & 0.54 \\
\hline HDSE & 97 & 3.9 & 3.5 & 2.9 & 0.16 & 0.54 \\
\hline
\end{tabular}


$0.7 \mu \mathrm{g} / \mathrm{mL}$ ) of three target compounds can meet the need of determination. This shows that UMAE method was feasible for the analysis of (-)-borneol, camphor and isoborneol in leaves.

Comparison of the proposed approach with the conventional method: The effect of material/water ratio on the yields of the crude extract in HDSE was shown in Fig. 2a and the effect of distilled aqueous solution volume on the yields of (-)-borneol, camphor and isoborneol in HDSE was also shown in Fig. 2b. After comparison, we found the optimal extraction conditions of HDSE: material/water ratio, 1:50; distilled aqueous solution volume, $300 \mathrm{~mL}$. A comparison was made between the proposed UMAE approach and the conventional HDSE method under optimal working conditions. The results in Table- 1 indicated that the precisions, stabilities, LODs and LOQs of UMAE and HDSE methods were similar in determination of three target compounds. The predecessors' researches showed that camphor and borneol contents of Flos Chrysanthemi indici, Chrysanthemum indici and Amomum villosum lour were determined by UAE-GC-FID, MAE-GC-FID, SD-GC-FID, PHWE-SPME-GC-MS and HS-SPME-GC-MS and the precisions of these methods were from $5.4 \%$ to $11.6 \%$, the recoveries were $86-95 \%^{12-15}$. As known from comparison of precision and recovery, the proposed UMAE method was close to the predecessors' research results. Although the recoveries of three target compounds determined by UMAE-GC-FID were worse than that of HDSE, the recoveries met the determination of three target compounds.

As shown in Table-2, the sample amount, water, solvent and power consumption of UMAE were less than that of HDSE. Moreover, the total extraction time (30 s) of UMAE was significantly shorter than that of HDSE (30 min) and it was also more rapid than MAE, UAE, SD, PHWE and HSSPME methods (from 4 min to $6 \mathrm{~h}$ ) recorded in the predecessors' researches ${ }^{12-15}$. The results indicated that the proposed UMAE procedure provided a rapid and effective approach for the extraction of the volatile compounds from plant leaves or other materials. The superiority of UMAE is mainly due to the excellent solution diffusion and energy transfer ability.

Quantitative analysis of Blumea balsamifera leaves by UMAE-GC-FID: B. balsamifera leaves from fourteen different times in harvest period (the interval time was half a month) were extracted by UMAE and HDSE techniques at the optimal conditions. The filtrate was analyzed by GC-FID. GC chromatogram of standard solution and B. balsamifera leaves sample with UMAE-GC-FID. The retention times of camphor, isoborneol, (-)-borneol and internal standard are 9.7, 11.8, 12.3 and $12.9 \mathrm{~min}$, respectively. According to the calibration curves, the concentrations of (-)-borneol, camphor and isoborneol in B. balsamifera leaves from different harvest times were calculated and the analytical results are shown in Table-3. There were no significant difference between the amounts of (-)borneol, camphor and isoborneol determined by two methods. Meanwhile, the variation of three target compounds in harvest period can be monitored.

The (-)-borneol content of leaves which were harvested during mid-October to next January were higher level, therefore this period was the optimal harvest time. Since (-)-borneol is a volatile compound and its loss is related with air temperature, less losses were found with lower air temperature. In the harvest period, the weather was cold so that there was more (-)-borneol to be contained in leaves. Isoborneol and (-)borneol are isomers, they have similar nature and their performances were consistent. Camphor content increased gradually from September to next March, which could be that the oxidation rate of (-)-borneol and isoborneol was greater than the volatilization rate of camphor in growth process. As known from results, the contents of camphor and isoborneol are very
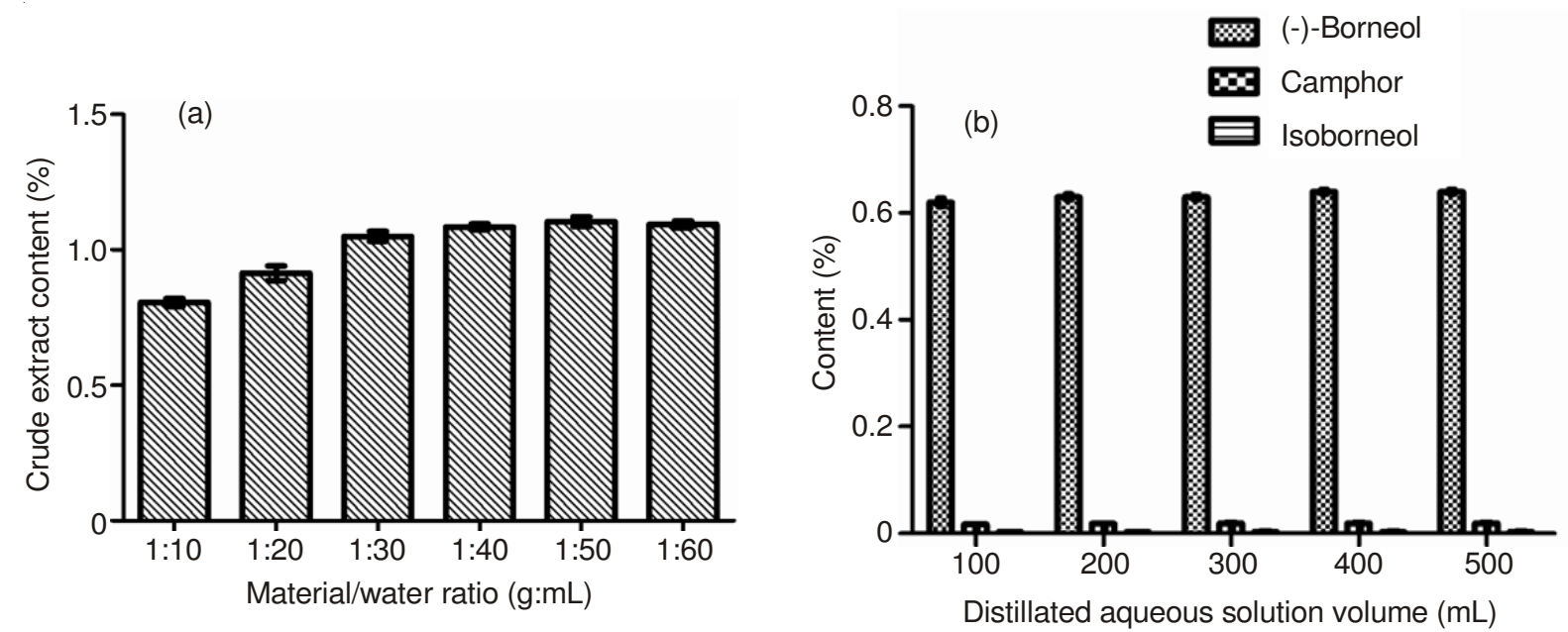

Fig. 2. Effect of material/water ratio (a) and distilled aqueous solution volume (b) on the HDSE extraction efficiencies of (-)-borneol, camphor and isoborneol in Blumea balsamifera leaves

TABLE-2

OPTIMUM EXTRACTION CONDITIONS OF TWO SAMPLING METHODS

\begin{tabular}{cccccc}
\hline Extraction Methods & Sample amount $(\mathrm{g})$ & Water volume $(\mathrm{mL})$ & Solvent volume $(\mathrm{mL})$ & Time & Power $(\mathrm{W})$ \\
\hline HDSE & 20 & 1000 & 300 & $30 \mathrm{~min}$ & 1000 \\
UMAE & 1 & 0 & 10 & $30 \mathrm{~s}$ & 150 \\
\hline
\end{tabular}




\begin{tabular}{|c|c|c|c|c|c|c|}
\hline \multirow{3}{*}{ Harvesting times } & \multicolumn{6}{|c|}{$\begin{array}{l}\text { TABLE-3 } \\
\text { THE CONCENTRATIONS OF (-)-BORNEOL, CAMPHOR AND ISOBORNEOL IN Blumea balsamifera } \\
\text { LEAVES HARVESTED FROM DIFFERENT HARVEST TIMES BY UMAE AND HDSE }\end{array}$} \\
\hline & \multicolumn{2}{|c|}{ (-)-Borneol (mg/g) } & \multicolumn{2}{|c|}{ Camphor (mg/g) } & \multicolumn{2}{|c|}{ Isoborneol (mg/g) } \\
\hline & UMAE & HDSE & UMAE & HDSE & UMAE & HDSE \\
\hline 15th Sep, 2011 & $6.3 \pm 0.1$ & $6.4 \pm 0.2$ & $0.23 \pm 0.02$ & $0.24 \pm 0.01$ & $0.023 \pm 0.001$ & $0.024 \pm 0.002$ \\
\hline 1st Oct, 2011 & $7.8 \pm 0.2$ & $7.8 \pm 0.3$ & $0.26 \pm 0.03$ & $0.25 \pm 0.01$ & $0.024 \pm 0.001$ & $0.025 \pm 0.001$ \\
\hline 15th Oct, 2011 & $9.1 \pm 0.2$ & $9.2 \pm 0.3$ & $0.31 \pm 0.02$ & $0.31 \pm 0.01$ & $0.032 \pm 0.002$ & $0.032 \pm 0.002$ \\
\hline 1st Nov, 2011 & $9.1 \pm 0.2$ & $9.1 \pm 0.3$ & $0.28 \pm 0.01$ & $0.29 \pm 0.01$ & $0.035 \pm 0.001$ & $0.036 \pm 0.001$ \\
\hline 15th Nov, 2011 & $9.1 \pm 0.2$ & $9.2 \pm 0.2$ & $0.38 \pm 0.03$ & $0.37 \pm 0.02$ & $0.036 \pm 0.001$ & $0.035 \pm 0.002$ \\
\hline 1st Dec, 2011 & $9.0 \pm 0.3$ & $9.1 \pm 0.3$ & $0.37 \pm 0.03$ & $0.36 \pm 0.02$ & $0.046 \pm 0.001$ & $0.047 \pm 0.002$ \\
\hline 15th Dec, 2011 & $9.1 \pm 0.3$ & $9.1 \pm 0.2$ & $0.36 \pm 0.02$ & $0.37 \pm 0.02$ & $0.045 \pm 0.001$ & $0.046 \pm 0.002$ \\
\hline 1st Jan, 2012 & $9.1 \pm 0.2$ & $9.2 \pm 0.2$ & $0.41 \pm 0.02$ & $0.42 \pm 0.02$ & $0.042 \pm 0.001$ & $0.043 \pm 0.002$ \\
\hline 15th Jan, 2012 & $8.8 \pm 0.2$ & $8.8 \pm 0.3$ & $0.41 \pm 0.03$ & $0.41 \pm 0.02$ & $0.041 \pm 0.001$ & $0.041 \pm 0.002$ \\
\hline 1st Feb, 2012 & $8.4 \pm 0.2$ & $8.3 \pm 0.2$ & $0.45 \pm 0.03$ & $0.47 \pm 0.01$ & $0.037 \pm 0.001$ & $0.039 \pm 0.002$ \\
\hline 15th Feb, 2012 & $8.0 \pm 0.1$ & $8.1 \pm 0.2$ & $0.57 \pm 0.05$ & $0.58 \pm 0.02$ & $0.042 \pm 0.001$ & $0.041 \pm 0.002$ \\
\hline 1st Mar, 2012 & $7.7 \pm 0.3$ & $7.8 \pm 0.2$ & $0.59 \pm 0.04$ & $0.58 \pm 0.02$ & $0.033 \pm 0.001$ & $0.034 \pm 0.001$ \\
\hline 15th Mar, 2012 & $7.3 \pm 0.1$ & $7.4 \pm 0.1$ & $0.58 \pm 0.03$ & $0.59 \pm 0.02$ & $0.031 \pm 0.001$ & $0.031 \pm 0.001$ \\
\hline 30th Mar, 2012 & $6.7 \pm 0.2$ & $6.9 \pm 0.2$ & $0.59 \pm 0.05$ & $0.61 \pm 0.03$ & $0.032 \pm 0.001$ & $0.031 \pm 0.001$ \\
\hline
\end{tabular}

low, two impurities hardly influenced (-)-borneol production. Therefore, Mastering (-)-borneol content and variation were critical to assess quality of $B$. balsamifera leaves.

\section{Conclusion}

In the present work, we successfully developed UMAEGC-FID for quantitative analysis of (-)-borneol, camphor and isoborneol in $B$. balsamifera leaves from different harvest times. Compared with hydrodistillation-solvent extraction, the proposed method needed a very little sample amount $(1 \mathrm{~g})$ and extraction time (30 s). The method required simple and inexpensive equipment and its operation is also simple. Therefore, UMAE-GC-FID is a simple and rapid approach to quantitative analysis of (-)-borneol, camphor and isoborneol in leaves. Through applying the method to determine (-)-borneol content of leaves harvested from fourteen different times and monitoring content variation, the period of mid-October to next January is the best harvest time and the highest yield of (-)-borneol will be obtained. The study demonstrates that UMAE-GC-FID is an alternative tool for B. balsamifera leaves quality monitoring and provides a preliminary reference information to natural (-)-borneol production.

\section{ACKNOWLEDGEMENTS}

This work was financially supported by Chinese Innovation Fund for Technology Based Firms (11C26215205838) and by Guizhou's Innovation Fund for Technology Based Firms (Qian Ke He Zi [2011] 5002).

\section{REFERENCES}

1. R. Lin, Flora Republicae Popularis Sinicae, vol. 75, Science Press Beijing, p. 19-20 (1979).
2. T.C. Zhu, Y.X. Wen, H.S. Wang and Y.L. Huang, Guihaia, 28, 141 (2008).

3. M.N.I. Bhuiyan, J.U. Chowdhury and J. Begum, Bangladesh J. Bot., 38, 109 (2009).

4. Chinese Pharmacopoeia Commission, Pharmacopoeia of The People's Republic of China, 2010 edition, vol. 1, China Medical Science Press, Beijing (2010).

5. Q. Shen, X. Li, W.J. Li and X.Y. Zhao, AAPS Pharm. Sci. Tech., 12, 1044 (2011).

6. W.R. Li, R.Y. Chen, L. Yang, T.L. Huang, Q.W. Xu, S.Q. Mi and N.-S. Wang, Eur. J. Drug Metab. Pharmacokinet., 37, 39 (2012).

7. E. Cherneva, V. Pavlovic, A. Smelcerovic and D. Yancheva, Molecules, 17, 10258 (2012).

8. J.C. Silva-Filho, N.N.P.M. Oliveira, D.D.R. Arcanjo, L.J. Quintans, S.C.H. Cavalcanti and M.R.V. Santos, Basic Clin. Pharmacol. Toxicol., 2, 177 (2012)

9. R. Liu, L. Zhang, X. Lan, L. Li, T.-T. Zhang, J.-H. Sun and G.-H. Du, Neuroscience, 176, 408 (2011).

10. X. Jiang, J. Pan, J. Si and B. Pan, Biomass Chem. Eng., 40, 20 (2006).

11. K.M. Lee, S.Y. Dai, T.J. Herrman and J.M.B. Musser, J. Chromatogr. B; Analyt. Technol. Biomed. Life Sci., 905, 133 (2012).

12. C.H. Deng, Y. Mao, N. Yao and X.M. Zhang, Anal. Chim. Acta, 575, 120 (2006).

13. L. Dong, J.Y. Wang, C.H. Deng and X.Z. Shen, J. Sep. Sci., 30, 86 (2007).

14. Q. Ye and C.H. Deng, Anal. Lett., 41, 2387 (2008).

15. Q. Ye and C.H. Deng, J. Chromatogr. Sci., 47, 287 (2009).

16. X.L. Cheng, J.Y. Wan, P. Li and L.W. Qi, J. Chromatogr. A, 1218, 5784 (2011).

17. H. Sereshti, Y. Izadmanesh and S. Samadi, J. Chromatogr. A, 1218, 4593 (2011).

18. S. Garcia-Salgado, M.A. Quijano and M.M. Bonilla, Anal. Chim. Acta, 714, 38 (2012).

19. Z.X. Lou, H.X. Wang, S. Zhu, S.W. Chen, M. Zhang and Z.P. Wang, Anal. Chim. Acta, 716, 32 (2012). 